\title{
How paper folds: bending with local constraints
}

\author{
Jemal Guven ${ }^{(1)}$ and Martin Michael Müller ${ }^{(2) * *}$ \\ (1) Instituto de Ciencias Nucleares, Universidad Nacional Autónoma de México \\ Apdo. Postal 70-543, 04510 México, D.F., MEXICO \\ (2) Max-Planck-Institut für Polymerforschung \\ Ackermannweg 10, 55128 Mainz, GERMANY
}

October 29, 2018

\begin{abstract}
A variational framework is introduced to describe how a surface bends when it is subject to local constraints on its geometry. This framework is applied to describe the patterns of a folded sheet of paper. The unstretchability of paper implies a constraint on the surface metric; bending is penalized by an energy quadratic in mean curvature. The local Lagrange multipliers enforcing the constraint are identified with a conserved tangential stress that couples to the extrinsic curvature of the sheet. The framework is illustrated by examining the deformation of a flat sheet into a generalized cone.
\end{abstract}

\section{Introduction}

There is a striking similarity in the patterns we observe in a crumpled ball of paper, a crushed aluminium can or, for that matter, the creases that develop on the back of one's shirt when one's back has been up against the wall a little too long. This is not an accident.

The relevant property that these materials share is that they offer resistance to tangential strain. This is captured geometrically in the statement that the metric, which determines distances between points on the surface (and thus is a quantity intrinsic to it), does not change under deformation: the only permissible deformations are isometries [1, 2, 3, 4]. Not only must the surface area remain fixed, right angles must remain right on the surface.

In general, the number of ways that a surface can bend without stretching is limited. In particular, if a flat sheet is bent in more than one direction it must stretch somewhere. This is a direct consequence of the geometrical fact-Gauss's inspired insight-that the product of the

\footnotetext{
${ }^{*}$ Current address: Laboratoire de Physique Statistique, Ecole Normale Supérieure, 24, rue Lhomond, 75231 Paris Cedex 05, FRANCE
} 
curvatures at any point on the surface depends only on the metric there [5]: two quantities that are defined extrinsically are constrained by the intrinsic geometry. In particular, if the surface is flat to start with, so that one or both of its curvatures vanishes, it must remain flat. However, the number of ways a surface can be flat is itself limited. It is either part of a cylinder, a cone or, more generally, a tangent developable surface (think Frank Gehry [6]), ruled by straight lines [1, 5, 7]. Any deformation of the surface is constrained locally by the fact that it must coincide with one of them.

The physically deformed surface is, of course, rarely any single one of these elementary flat surfaces. This is because the forces applied to the surface usually oblige it to fold along more than one direction somewhere. It turns out, however, that the most energy effective way to adjust to such forces is by confining the regions where stretching occurs within a series of sharp peaks and ridges [8, 9]. A tangent plane cannot be drawn at these singular points; the curvatures will generally diverge. The complete deformed surface will form a quilt of irregularly shaped flat patches meeting along the boundaries that these points provide. As described by Cerda and Mahadevan they provide the hinges about which the flat patches may flap [10]. Once such singular points are established, however, irreversible damage will be done to the surface: iron out the creases we may but the paper will remain scored, the can scarred and, perhaps this is stretching the analogy, shirts do become threadbare.

A theory which describes the detailed internal structure of this network of peaks and ridges will be complicated and it will depend on the detailed material properties of paper. An approximation, reliable for small strains, is provided by the Föppl-von Karman equations [11]. The length scale of these regions is on the order of the thickness of the sheet; viewed from outside, however, this internal structure is irrelevant: peaks become points and ridges can be treated as curves along which boundary conditions are set on the flat surface; the only surviving material parameter in the physical description of the sheet is a modulus characterizing its stiffness. The important point is that on these longer length scales the only relevant degrees of freedom are the geometrical ones; how the surface bends becomes an essentially geometrical problem, a fact that tends to get lost when it is treated from a continuum mechanical point of view. In this paper we would like to explore this geometrical approach to the problem.

The bending energy of a surface is deceptively simple; modulo a topological energy there is a single quadratic in curvature associated with a surface [12, 13]. However, determining the bending patterns of a surface when bending alone is penalized provides a subtle non-linear problem in itself and there are few useful analytical results. The problem we are interested in addressing has an added complication: there are local geometrical constraints on the surface; the metric is fixed.

When the constraints are global it is straightforward to enforce them in the minimization of the energy by introducing Lagrange multipliers. If the enclosed volume and the area are prescribed, these multipliers are identified with the equilibrium pressure and tension: they are global. This is a situation that is familiar (in the context of fluid membranes see, for example, [14]). With 
local constraints these multipliers get replaced by local fields with their own conservation laws reflecting the constraint.

In this paper we will set up a variational framework to describe the equilibrium shapes assumed by a surface subject to local constraints. In equilibrium, we will show that the multipliers are identified with a conserved tangential surface stress. The constraints fixing the metric thus set up tangential stresses within the sheet. These add to the stresses associated with bending. They couple linearly to the extrinsic curvature in the shape equation describing the equilibrium configuration.

We will illustrate the framework by examining the deformation of a flat sheet into a generalized cone [9]. This is the simplest flat deformation of a plane exhibiting a localized distribution of energy. Our focus will be on the stresses set up in the cone rather than the geometrical details of the configuration itself. We will examine in detail how the different contributions to the stress conspire to transmit force and torque along closed curves on the cone. In particular, we will show how our framework can be applied to two physically interesting setups that have been discussed in the literature. The first of these considers the depression of a circular sheet into a circular frame by application of a point force to its center [10]. Neither the sheet nor the frame needs to be circular. We will show that the consistent coupling to curvature is enough to completely determine the radial behavior of the stress tangent to a sphere centered on the apex of the cone. The radial behavior of the remaining projections of the stress tensor is then completely determined by the conservation law. We will show that additional off-diagonal stresses, that have not been studied previously, are consistent with the conical geometry. The second example considers the 'draping' patterns assumed by a sheet supported at a point that falls under the influence of gravity [15]. Additional stress fields are now needed to counteract the effects of gravity which would tend to unflatten the disc. We show, however, that they

are also completely determined by the consistent coupling to curvature. Remarkably, the shape equation we obtain assumes a universal form, with or without gravity; the physical details of the problem enter the equation only through the stress tangent to the sphere associated with the constraint. In particular, with gravity, this equation differs from its (technically inaccurate) counterpart obtained by pre-averaging over the radial direction.

\section{Bending a constrained sheet}

We are interested in determining the configurations that minimize the bending energy of an initially flat unstretchable surface under the influence of some set of external forces or constraints. This might be gravity, forces that act locally or the confinement of the surface within a fixed volume. We will first examine the problem without the complications introduced by gravity. 
The bending energy associated with the configuration $\mathbf{X}$ is given by

$$
H[\mathbf{X}]=\frac{1}{2} \int d A K^{2},
$$

where $K=C_{1}+C_{2}$ is the sum of the principal curvatures (twice the mean curvature), and $d A$ is the area element induced on the surface (our notation is summarized in [16]). We have set the rigidity modulus to be one.

Bending must be an isometry: not only does the geometry of the sheet remain flat almost everywhere when it is bent, it also resists shear and stretching. Thus it is not sufficient to demand that the Gaussian curvature vanishes; one needs to fix the metric itself. The bending energy $H[\mathbf{X}]$ defined by Eq. (1) needs to be minimized subject to this constraint. The framework will also need to accommodate discontinuities and singularities which arise due to global obstructions on this requirement.

Distances on the surface are described by the metric, $g_{a b}=\mathbf{e}_{a} \cdot \mathbf{e}_{b}$, where $\mathbf{e}_{a}=\partial_{a} \mathbf{X}, a=1,2$, are the two tangent vectors adopted to the particular parametrization of the surface. In a sheet of paper the metric is fixed, $g_{a b}^{(0)}$ say. We thus construct a functional to reflect this constraint

$$
H_{C}[\mathbf{X}]=H[\mathbf{X}]-\frac{1}{2} \int d A T^{a b}\left(u^{a}\right)\left(g_{a b}-g_{a b}^{(0)}\right) .
$$

Here $T^{a b}\left(u^{a}\right)$ is a set of local Lagrange multipliers implementing the constraint on the metric. Both $g_{a b}$ and $g_{a b}^{(0)}$ as well as the multipliers $T^{a b}$ form tensors, so that the trace appearing in Eq. (2) is a scalar. Thus, by construction, the functional $H_{C}$ is independent of the parametrization.

With the constraint in place, it is possible to introduce a deformation $\mathbf{X} \rightarrow \mathbf{X}+\delta \mathbf{X}$ in the shape without needing to worry if the metric is behaving.

In the absence of external sources of stress, the constrained equilibrium may be expressed as the conservation law [17]

$$
\nabla_{a} \mathbf{f}^{a}=0,
$$

where the stress tensor $\mathbf{f}^{a}$ is a sum of two terms:

$$
\mathbf{f}^{a}=\mathbf{f}_{0}^{a}+T^{a b} \mathbf{e}_{b} .
$$

The bending contribution is given by (for our notation see [16])

$$
\mathbf{f}_{0}^{a}=K\left(K^{a b}-\frac{1}{2} g^{a b} K\right) \mathbf{e}_{b}-\left(\nabla^{a} K\right) \mathbf{n} .
$$

There is a particularly direct way to derive these equations. It involves a natural extension of an approach to the equilibrium of fluid membranes using a set of auxiliary variables introduced by one of the authors [18]. 
The constraint adds a tangential stress proportional to the multiplier $T^{a b}$; tension will be introduced in the surface whenever its action is antagonistic to bending. This tension will generally be inhomogeneous and anisotropic.

The 'shape equation' is given by the normal projection of the conservation law (3):

$$
\mathcal{E}=\mathcal{E}_{0}-K_{a b} T^{a b}=0
$$

where

$$
\mathcal{E}_{0}=-\nabla^{2} K+\frac{1}{2} K\left(K^{2}-2 K_{a b} K^{a b}\right) .
$$

Here $\nabla^{2}$ is the Laplacian on the surface constructed with the induced metric. The stress $T^{a b}$ couples linearly to the extrinsic curvature in Eq. (6).

The tangential component of the conservation law (3) is the statement that $T^{a b}$ itself is conserved,

$$
\nabla_{a} T^{a b}=0 .
$$

The bending energy does not enter this equation. It is an intrinsic statement involving the intrinsic geometry through the covariant derivative. We note that $T^{a b}$ involves three degrees of freedom; this is the correct number of components to pin-down the three components of the metric. From a field theoretical point of view it is also possible to view the two constraints provided by Eq. (8) abstractly as a requirement for consistency on the $T^{a b}$ implied by reparametrization invariance [17]. Note that the tangential part of $\mathbf{f}_{0}$ defined by Eq. (5), unlike $T^{a b}$, is not conserved.

It is worth pointing out that this variational approach to bending with a local constraint is more direct than its continuum mechanical counterpart. One does not need to invoke any adapted system of coordinates. One also sidesteps the need to introduce constitutive relations; once the energy function is given, the relationship between stress and strain follows.

One now needs to solve the coupled system of equations (6) and (8) subject to appropriate boundary conditions. Eq. (6) will pick up a source if external forces are operating on the sheet. In addition, the geometry will typically display geometrical singularities where Eq. (8) will pick up a distributional source. We will apply this framework to the conical geometry in the next section.

It is also instructive to examine how this setup would be different had we fixed only the Gaussian curvature. This is addressed in appendix $\mathrm{A}$. The relevant extension of our framework to handle the situation in which the initial surface is not flat is a straightforward generalization. 


\section{Folding a cone}

\subsection{Geometry of a Developable Cone}

We begin with a brief review of the conical geometry described by Ben Amar and Pomeau [9]. For further details see [10].

A cone possesses a natural parametric representation in terms of a closed curve $\Gamma: s \rightarrow \mathbf{u}(s)$ on the unit sphere. Let $s$ be arc-length along this curve and $r$ be the distance from the origin along the ray pointing in the direction $\mathbf{u}(s)$ (see Fig. 1). The image of the mapping

$$
(r, s) \rightarrow \mathbf{X}(r, s)=r \mathbf{u}(s)
$$

describes a cone. We will suppose that this cone is the result of an isometric deformation of a planar disc (not necessarily circular) of radius $R(s)$. The length of $\Gamma$ is then fixed at $L=2 \pi$. This means that the closed curve which represents the cone lives always within a single hemisphere. A flat planar disc is represented by a great circle. These are the only circular configurations consistent with the constraint on the length. If the disc is circular of radius $R$ and the apex coincides with its center, the maximum value of $r$ is given by $R$.

The tangent vectors to the cone adapted to this parametrization are $\mathbf{u}$ itself and $\mathbf{t}=\mathbf{u}^{\prime}$, where prime denotes a derivative with respect to $s$. Note that $\mathbf{u} \cdot \mathbf{u}^{\prime}=0$ so that the induced metric on the surface is specified by the flat line element

$$
d l^{2}=d r^{2}+r^{2} d s^{2}
$$

Let $\mathbf{n}=\mathbf{u} \times \mathbf{t}$ denote the normal to the surface. The extrinsic curvature tensor is given by

$$
K_{a b}=r\left(\begin{array}{cc}
0 & 0 \\
0 & \kappa
\end{array}\right) \text {, }
$$

where

$$
\kappa=-\mathbf{n} \cdot \mathbf{t}^{\prime}=-(\mathbf{u} \times \mathbf{t}) \cdot \mathbf{t}^{\prime}=-\mathbf{u} \cdot\left(\mathbf{t} \times \mathbf{t}^{\prime}\right) .
$$

The flat direction is along $\mathbf{u}$. The Gaussian curvature $K_{\mathrm{G}}=\operatorname{det}\left(K_{a}^{b}\right)$ vanishes. The curvature $K=\kappa / r$ diverges at the apex of the cone. This translates into a logarithmic divergence in the bending energy at this point.

The three vectors $\mathbf{u}, \mathbf{t}$ and $\mathbf{n}$ form a right-handed basis. Given $\kappa$, the curve can be reconstructed using the structure equations

$$
\begin{aligned}
\mathbf{u}^{\prime} & =\mathbf{t} \\
\mathbf{t}^{\prime} & =-\kappa \mathbf{n}-\mathbf{u} \\
\mathbf{n}^{\prime} & =\kappa \mathbf{t} .
\end{aligned}
$$



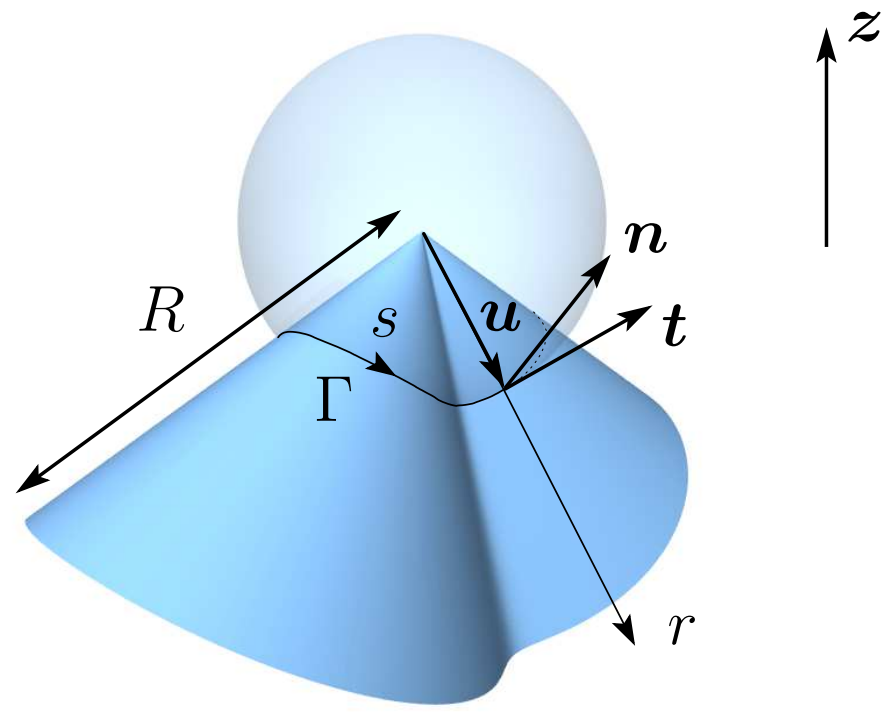

Figure 1: The cone geometry. A closed curve $\Gamma(s)$ on the unit sphere is sufficient to describe the surface completely.

Note that the vectors $\mathbf{u}$ and $\mathbf{n}$ are in general not equal to the normal and binormal vector of the curve [7]. Thus $\kappa$ should not be mistaken for the Frenet curvature, $\kappa_{F}$. It is simple, however, to show that the two are related by $\kappa_{F}^{2}=\kappa^{2}+1$.

\subsection{The cone as a constrained minimum}

We have seen that cones can be identified with curves on a unit sphere. As described in appendix $\mathrm{B}$, by integrating over the radial direction $H$ becomes a functional of curves on this sphere. As such, one could vary the Hamiltonian with respect to these curves to obtain an Euler-Lagrange equation which is satisfied by the curves which minimize the energy. This curve is then identified with the equilibrium shape of the conical surface. While this approach yields the correct shape equation in this case, it does not always provide the correct answer. As we will see, variation of the Hamiltonian and restriction of the geometry (integrating out the radial dependence in the conical geometry) do not commute in general even when the disc is circular. Even in this case, however, it does not possess the scope to provide a complete analysis of the problem.

We will instead apply our general framework to study the conical equilibrium. We first confirm 
that cones are in fact equilibrium geometries. It is straightforward to show that

$$
\mathcal{E}_{0}=-\nabla^{2} K+\frac{1}{2} K\left(K^{2}-2 K_{a b} K^{a b}\right)=-\frac{1}{r^{3}}\left(\kappa^{\prime \prime}+\frac{1}{2} \kappa^{3}+\kappa\right) .
$$

Note the appearance of the term linear in $\kappa$ originating in the radial part of the Laplacian. The shape equation thus reads

$$
-\frac{1}{r^{3}}\left(\kappa^{\prime \prime}+\frac{1}{2} \kappa^{3}+\kappa\right)-\kappa r T^{s s}=0
$$

Only the stress tangential to the generating curve on the unit sphere contributes.

The shape equation describing the conical equilibrium must hold for all $r$. Indeed, consistency requires that $r^{4} T^{s s}$ depends only on $s$. Let us write $r^{4} T^{s s}=-C_{\|}(s)$.

At this point it is also convenient to replace the components of the stress tensor $T^{a b}$ by the corresponding scalar quantities representing its projections along the directions tangential to the sphere and along its radius, $\mathbf{t}$ and $\mathbf{u}: T_{\|}=t_{a} t_{b} T^{a b}, T_{\| \perp}=t_{a} u_{b} T^{a b}$, and $T_{\perp}=u_{a} u_{b} T^{a b} 1$ In particular, the tangential stress

$$
T_{\|}=t_{s} t_{s} T^{s s}=r^{2} T^{s s}=-C_{\|}(s) \frac{1}{r^{2}}
$$

diverges as $r^{-2}$. We see immediately that the consistency of the shape equation with a conical geometry places a very strong constraint on the admissible form of $T_{\|}$, determining completely its radial dependence.

Let us now examine the conservation law for $T^{a b}$ to see what it tells us about $T_{\|}, T_{\perp}$ and $T_{\| \perp}$. The tangential and radial projections of Eq. (8) read:

$$
\begin{aligned}
\frac{1}{r} T_{\|}^{\prime}+\frac{\partial}{\partial r} T_{\| \perp}+\frac{2}{r} T_{\| \perp} & =0 ; \\
\frac{\partial T_{\perp}}{\partial r}+\frac{1}{r}\left(T_{\perp}-T_{\|}\right)+\frac{1}{r} T_{\| \perp}^{\prime} & =0 .
\end{aligned}
$$

Often the symmetry of the problem will imply $T_{\| \perp}=0$ so that, modulo Eq. (17), $C_{\|}$is a constant independent of $s$. If the circular frame discussed in [10] is replaced by one that is irregular, however, the balance of forces on the frame will require $T_{\| \perp} \neq 0$.

Remarkably, when $C_{\|}$is constant, as it will if the disc is circular, the shape equation assumes exactly the same form as the Euler-Lagrange equation for planar Euler Elastica with tension $\sigma \propto C_{\|}-1$ and $\kappa$ in place of the Frenet curvature, $\kappa_{F}$.

\footnotetext{
${ }^{1}$ With respect to the adapted coordinate system, $t^{a}=\left(0, r^{-1}\right)$ and $u^{a}=(1,0)$ so that $t_{a}=(0, r)$ and $u_{a}=(1,0)$.
} 
The Euler-Lagrange equation for planar Euler Elastica is completely integrable for $\kappa$ allowing the cone to be reconstructed using the structure equations. The tension in this fictitious Elastica can be either positive or negative, depending on the boundary conditions. This should be compared to the results of Cerda and Mahadevan who do not admit negative values [10]. Note that if $\kappa_{F}$ is used in place of $\kappa$ the equation is different. The shape does not correspond to Euler Elastica on a sphere [19].

More generally, the off-diagonal constraining stress is given by

$$
T_{\| \perp}=C_{\|}(s)^{\prime} \frac{\ln r}{r^{2}}+\frac{C_{\| \perp}(s)}{r^{2}},
$$

where $C_{\| \perp}$ is some other function of $s$. In a moment, we will see that if $C_{\| \perp}(s)$ is non-vanishing, it will play a role in the balance of torques on the cone.

The second conservation law (18) identifies the radial scalar $T_{\perp}$ as a sum of five terms

$$
T_{\perp}=C_{\|}(s)^{\prime \prime}\left(\frac{\ln r}{r^{2}}+\frac{1}{r^{2}}\right)+\frac{C_{\|}(s)}{r^{2}}-\frac{C_{\| \perp}(s)^{\prime}}{r^{2}}+\frac{C_{\perp}(s)}{r} .
$$

In particular, if $C_{\|}$is constant and $C_{\| \perp}=0$,

$$
T_{\perp}=\frac{C_{\|}}{r^{2}}+\frac{C_{\perp}(s)}{r} .
$$

The $r^{-2}$ behavior term is slaved to $T_{\|}$. This part of the stress tensor $T^{a b}$ is traceless: $g^{a b} T_{a b}=0$, a property that can be tracked back to the scale invariance of the bending energy. The $r^{-1}$ behavior indicates the existence of a source term associated with external forces.

It is worth emphasizing the following points: the stress associated with the constraint on the metric is explicitly anisotropic and inhomogeneous. It is not necessarily diagonal with respect to the orthonormal frame adapted to the cone. While it is not monotonic, it does vanish asymptotically.

It is also useful to possess explicit expressions for the surface components of the stress, $\mathbf{f}_{\|}=t_{a} \mathbf{f}^{a}$ and $\mathbf{f}_{\perp}=u_{a} \mathbf{f}^{a}$ representing the force per unit length transmitted across curves of constant $s$ and $r$ respectively [20]. A straightforward calculation gives

$$
\begin{aligned}
\mathbf{f}_{\|} & =\frac{1}{r^{2}}\left[\left(\frac{1}{2} \kappa^{2}+r^{2} T_{\|}\right) \mathbf{t}+r^{2} T_{\| \perp} \mathbf{u}-\kappa^{\prime} \mathbf{n}\right] \\
\mathbf{f}_{\perp} & =\frac{1}{r^{2}}\left[\left(-\frac{1}{2} \kappa^{2}+r^{2} T_{\perp}\right) \mathbf{u}+r^{2} T_{\| \perp} \mathbf{t}+\kappa \mathbf{n}\right] .
\end{aligned}
$$

As a check of consistency, note that the divergence may be expressed as

$$
\nabla_{a} \mathbf{f}^{a}=\frac{1}{r} \mathbf{f}_{\|}^{\prime}+\frac{\partial \mathbf{f}_{\perp}}{\partial r}+\frac{1}{r} \mathbf{f}_{\perp}
$$


In particular, it is simple to confirm that the projections of Eq. (24) along $\mathbf{n}, \mathbf{t}$ and $\mathbf{u}$ reproduce Eqs. (15), (17) and (18) respectively.

\section{Force and torque balance at constant $r$}

Now look at the balance of forces along a closed curve $\Gamma_{r}$ of constant $r$. The physical arc-length is $d \tau=r d s$. The normal to this contour pointing away from the apex is the vector $\mathbf{u}$. Then the total force transmitted across the curve is given by 2

$$
\begin{aligned}
\mathbf{F}=\oint_{\Gamma_{r}} d \tau \mathbf{f}_{\perp} & =\frac{1}{r} \oint d s\left(\kappa \mathbf{n}-\frac{1}{2} \kappa^{2} \mathbf{u}\right)+r \oint d s T_{\perp} \mathbf{u}+r \oint d s T_{\| \perp} \mathbf{t} \\
& =-\frac{1}{r} \oint d s\left(1+\frac{1}{2} \kappa^{2}\right) \mathbf{u}+r \oint d s T_{\perp} \mathbf{u}+r \oint d s T_{\| \perp} \mathbf{t}
\end{aligned}
$$

where we have used Eq. (23) on the first line and Eq. (13b) on the second. A much more transparent expression is possible in terms of the solution of the conservation law Eq. (8) for $T^{a b}$. Look at the integral along any closed curve of the Euler-Lagrange derivative $\mathcal{E}$, weighted by $\mathbf{n}$. In equilibrium,

$$
\oint d s\left(\kappa^{\prime \prime}+\frac{1}{2} \kappa^{3}+\kappa+\kappa r^{2} T_{\|}\right) \mathbf{n}=0 .
$$

Now, performing an integration by parts on the first term and using Eq. (13c), we obtain

$$
\oint d s \kappa^{\prime \prime} \mathbf{n}=-\oint d s \kappa \kappa^{\prime} \mathbf{t} .
$$

Furthermore, using Eq. (13b) to express $\mathbf{n}$ as a linear combination of $\mathbf{u}$ and $\mathbf{t}^{\prime}$, we obtain

$$
\begin{aligned}
\oint d s\left(\frac{1}{2} \kappa^{3}+\kappa+\kappa r^{2} T_{\|}\right) \mathbf{n}= & -\oint d s\left(\frac{1}{2} \kappa^{2}+1+r^{2} T_{\|}\right)\left(\mathbf{t}^{\prime}+\mathbf{u}\right) \\
= & \oint d s \kappa \kappa^{\prime} \mathbf{t}+r^{2} \oint d s T_{\|}^{\prime} \mathbf{t} \\
& -\oint d s\left(\frac{1}{2} \kappa^{2}+1+r^{2} T_{\|}\right) \mathbf{u} .
\end{aligned}
$$

Thus, the tangential stress is constrained as follows on a closed cone:

$$
\oint d s\left(1+\frac{1}{2} \kappa^{2}\right) \mathbf{u}+r^{2} \oint d s T_{\|} \mathbf{u}-r^{2} \oint d s T_{\|}^{\prime} \mathbf{t}=0 .
$$

\footnotetext{
${ }^{2}$ Any closed curve homotopic to $\Gamma_{r}$ can be used to determine the force replacing $\mathbf{f}_{\perp}$ by $l^{a} \mathbf{f}_{a}$, where $\mathbf{l}=l^{a} \mathbf{e}_{a}$ is the normal to the curve on the cone pointing towards increasing $r$.
} 
Modulo this identity we can finally write the total force in terms of the function $C_{\perp}$ appearing in Eq. (20) as

$$
\mathbf{F}=r \oint_{\Gamma_{r}} d s\left[\left(T_{\perp}+T_{\|}\right) \mathbf{u}+\left(T_{\| \perp}-T_{\|}^{\prime}\right) \mathbf{t}\right]=\oint_{\Gamma_{r}} d s C_{\perp}(s) \mathbf{u}
$$

where we have used the expressions for the projections of the stress tensor (16), (19) and (20) consistent with the shape equation and the conservation of $T^{a b}$. The line integration does not depend on $r$. If an external force is acting, the term proportional to $r^{-1}$ in $T_{\perp}$ does not vanish. If a force $\mathbf{F}$ is applied at the apex of a cone supported on a frame, $C_{\perp}(s)$ is the distribution of forces on the rim of this frame needed to counterbalance it. Notice that the tangential and the radial projections of the conservation law can be written as

$$
\nabla_{a} T^{a b}=\left[C_{\perp}(s) u^{b}+C_{\| \perp}(s) t^{b}\right] \frac{\delta(r)}{r} .
$$

In exactly the same way, we can consider the torque. The conserved torque tensor is given by [17, 22]

$$
\mathbf{m}^{a}=\mathbf{X} \times \mathbf{f}^{a}+K g^{a b} \mathbf{e}_{b} \times \mathbf{n}
$$

and the total torque about the origin can be written as:

$$
\mathbf{M}=\oint_{\Gamma_{r}} \mathrm{~d} \tau u_{a} \mathbf{m}^{a}
$$

Thus

$$
\begin{aligned}
\mathbf{M} & =r \oint_{\Gamma_{r}} d s\left[\left(r \mathbf{u} \times \mathbf{f}_{\perp}\right)+K \mathbf{u} \times \mathbf{n}\right] \\
& =-2 \oint_{\Gamma_{r}} d s \kappa \mathbf{t}+r^{2} \oint_{\Gamma_{r}} d s T_{\| \perp} \mathbf{n} \\
& =\oint_{\Gamma_{r}} d s C_{\| \perp}(s) \mathbf{n} .
\end{aligned}
$$

The integrated contribution from $\mathrm{f}_{0}^{a}$ vanishes on account of the structure $\mathrm{Eq}$. (13c) and the closure of $\Gamma_{r}$. Furthermore, the term proportional to $\ln r / r^{2}$ in $T_{\| \perp}$ [see Eq. (19)] does not contribute to the torque as one can show by integrating the Euler-Lagrange derivative (15) weighted by $\mathrm{t}$ along a closed curve.

Eq. (34) implies that the cone geometry may provide an equilibrium when an external torque is applied. In that case, the off-diagonal stresses do not vanish and the value of the torque is captured by $C_{\| \perp}(s)$. 


\section{Brought down by gravity}

Suppose that the sheet is held at a point and allowed to fall under gravity. The bending energy is minimized by the planar disc; gravitational potential energy is minimized by a sheet that hangs vertically; as described in [15], the competition between the two gives rise to draping patterns.

If the weight of the sheet is taken into account, its gravitational potential energy is given by

$$
H_{g}[\mathbf{X}]=-\rho g \int d A \mathbf{X} \cdot \mathbf{z},
$$

where $\rho$ is the density of the sheet and $g$ is the acceleration due of gravity. To counterbalance the weight of the sheet an external force $\mathbf{F}_{\text {ext }}$ in the $\mathbf{z}$-direction has to be applied (see Fig. 11). This force acts at the apex of the cone (see previous section).

Under the deformation, $\mathbf{X} \rightarrow \mathbf{X}+\delta \mathbf{X}$, the variation of $H_{g}$ is given by

$$
\delta H_{g}=-\rho g \int d A(K \mathbf{X} \cdot \mathbf{z}+\mathbf{n} \cdot \mathbf{z}) \mathbf{n} \cdot \delta \mathbf{X} .
$$

The effect is to add a normal source to the conservation law (3):

$$
\begin{aligned}
\nabla_{a} \mathbf{f}^{a} & =\rho g(K \mathbf{X} \cdot \mathbf{z}+\mathbf{n} \cdot \mathbf{z}) \mathbf{n} \\
& =\rho g(\kappa \mathbf{u} \cdot \mathbf{z}+\mathbf{n} \cdot \mathbf{z}) \mathbf{n}=j(s) \mathbf{n} .
\end{aligned}
$$

The Euler-Lagrange equation (15) is replaced by the equation

$$
-\frac{1}{r^{3}}\left(\kappa^{\prime \prime}+\frac{1}{2} \kappa^{3}+\kappa\right)-\frac{\kappa}{r} T_{\|}=j(s) .
$$

This differs from the result that Cerda et al. have derived. They average over the radial direction [15].

As before, consistency will place a constraint on $T_{\|}$. However, we now require that 3

$$
T_{\|}=-\frac{C_{\|}(s)}{r^{2}}-\frac{j}{\kappa} r=T_{\|}^{\mathrm{g}=0}-\frac{j}{\kappa} r
$$

Even in the regime where the analysis provided in appendix $B$ is valid, with gravity we begin to see how such an approach breaks down. The pre-averaging over $r$ provides a different (and incorrect) shape equation.

\footnotetext{
${ }^{3}$ In the following, all variables with an upper index $g=0$ refer to the case without gravity.
} 
The conservation law (8) for $T^{a b}$ is unchanged as gravity only contributes a normal source. However, because of Eq. (39), in place of Eqs. (19) and (20) we have

$$
T_{\| \perp}=T_{\| \perp}^{\mathrm{g}=0}+\left(\frac{j}{\kappa}\right)^{\prime} \frac{r}{3}
$$

and

$$
T_{\perp}=T_{\perp}^{\mathrm{g}=0}-\left[\frac{j}{\kappa}+\frac{1}{3}\left(\frac{j}{\kappa}\right)^{\prime \prime}\right] \frac{r}{2} .
$$

The force transmitted across a curve of constant $r$ is then given by

$$
\begin{aligned}
& \mathbf{F}=\oint_{\Gamma_{r}} d \tau \mathbf{f}_{\perp}=r \oint_{\Gamma_{r}} d s\left[\left(T_{\perp}+T_{\|}^{\mathrm{g}=0}\right) \mathbf{u}+\left(T_{\| \perp}-T_{\|}^{\prime} \mathrm{g}=0\right) \mathbf{t}\right] \\
& \stackrel{30}{=} \oint_{\Gamma_{r}} d s C_{\perp}(s) \mathbf{u}+r^{2} \oint_{\Gamma_{r}} d s\left\{\frac{1}{2}\left[\frac{j}{\kappa}+\frac{1}{3}\left(\frac{j}{\kappa}\right)^{\prime \prime}\right](-\mathbf{u})+\frac{1}{3}\left(\frac{j}{\kappa}\right)^{\prime} \mathbf{t}\right\} \\
& \stackrel{13 \mathrm{~b}}{=} \oint_{\Gamma_{r}} d s C_{\perp}(s) \mathbf{u}+\frac{r^{2}}{2} \oint_{\Gamma_{r}} d s j \mathbf{n}-\frac{r^{2}}{6} \oint_{\Gamma_{r}} d s\left[\left(\frac{j}{\kappa}\right)^{\prime} \mathbf{u}\right]^{\prime}=-\mathbf{F}_{\text {ext }}+\int_{\Sigma_{r}} \mathrm{~d} A j \mathbf{n},
\end{aligned}
$$

where $\Sigma_{r}$ is that part of the surface which is enclosed by the curve $\Gamma_{r}$. Note that the line integration does now depend on $r$ but for good reason: the surface below the curve $\Gamma_{r}$ exerts the force $\mathbf{F}$ on $\Gamma_{r}$ due to gravity. Its absolute value decreases quadratically for increasing $r$ and vanishes for $r=R$ as expected.

The torque about the origin is now given by

$$
\mathbf{M}=r^{2} \oint_{\Gamma_{r}} d s T_{\| \perp} \mathbf{n} \stackrel{40}{=}=\mathbf{M}^{\mathrm{g}=0}+r^{2} \oint_{\Gamma_{r}} d s\left(\frac{j}{\kappa}\right)^{\prime} \frac{r}{3} \mathbf{n}=\mathbf{M}^{\mathrm{g}=0}-\int_{\Sigma_{r}} d A r j \mathbf{t}
$$

The last term due to gravity can also be written as $\int_{\Sigma_{r}} d A[\mathbf{X} \times(j \mathbf{n})]$, again exactly as one would expect.

\section{Discussion}

In this paper we have introduced a geometrical framework to examine the bending of an (unstretchable) sheet of paper. We have illustrated the viability of this framework by examining the conical deformations of a planar sheet. In particular, we have examined in some detail the distribution of stresses within the sheet associated both with bending and with the constraint and how they conspire to transmit the external forces and torques acting on the sheet.

Various characteristic patterns are associated with this geometry: the radial dependence of the stress is constrained strongly by the geometry; how it varies along the spherical generating curves will depend on the specific boundary conditions associated with the external forces acting on the 
sheet. We have also shown how the presence of bulk forces such as gravity are accommodated within this framework. In the latter case, we point out the pitfalls of prematurely constraining the geometry within the variational principle.

We have not attempted to discuss the physical details within the ridges and peaks where the elastic approximation breaks down. Various elements of our framework are, however, likely to play a role in any refinement of the model which does address this physics.

We have limited our discussion of the application of our framework to conical shapes; this is because they are relatively simple not because they are the only interesting configurations. It would be interesting (and it should be straightforward) to examine the Möbius strip geometry described recently by Starostin and van der Heijden within our framework [23].

It is appropriate to think of a cone as a kind of elementary deformation of a planar sheet in which the energy is localized. A generic patch of flat surface, however, will be described by a tangent developable surface. Such a surface is singular along a certain curve: its edge of regression which will itself generally exhibit singularities [5, 7]. The flat directions are generated by the tangent to this curve. Typically, we do not see this curve when we fold paper. The patch gets truncated by a ridge or we run off the sheet before the corresponding generating curve is reached. However, the folded sheet is, in principal, completely described by this set of curves. This description will be the subject of a subsequent publication [24].

\section{Acknowledgments}

We thank Riccardo Capovilla for getting us interested in this problem. We have benefitted from conversations with him and Markus Deserno. Timo Schürg gave us some valuable tips on how to use POV-Ray. Partial support from CONACyT grant 51111 as well as DGAPA PAPIIT grant IN119206-3 is acknowledged.

\section{A Fixing Gaussian curvature}

Let us examine the consequences of fixing the Gaussian curvature instead of the metric. We thus construct the constrained functional

$$
H_{C}[\mathbf{X}]=H[\mathbf{X}]+\int d A W\left(u^{a}\right) K_{\mathrm{G}}
$$

where $K_{\mathrm{G}}$ is the Gaussian curvature [16]. Here $W\left(u^{a}\right)$ is a local Lagrange multiplier constraining $K_{\mathrm{G}}$ to vanish. Analogous functionals can be defined for a constraint on any other geometrical scalar. 
The constrained equilibrium may again be expressed as a conservation law of the form (3). The stress tensor $\mathbf{f}^{a}$ is again given by an expression of the form (4) with an additional tangential stress $T^{a b}$. However, this stress is now determined completely by the multiplier $W$,

$$
T^{a b}=\frac{1}{2}\left(\nabla^{a} \nabla^{b} W-g^{a b} \nabla^{2} W\right) .
$$

The constraint again introduces tension in the surface but, this time, with a single degree of freedom.

It is appropriate to point out that there is an ambiguity inherent in the definition of the stress tensor $\mathbf{f}^{a}$. This ambiguity is due, in part, to the Gauss-Codazzi integrability condition which connects the intrinsic and extrinsic geometry [26]. If the variations are treated intrinsically, one obtains the expression we have written down for the stress; if, however, they are treated extrinsically an apparently very different answer is obtained. The two are entirely consistent; they differ only by a conserved null stress that does not transmit forces. The relevant calculations have appeared in a different context (see, for example, [18]).

Whereas the conservation of $T^{a b}$ is an important constraint when we fix the metric, here it is simple to confirm that $T^{a b}$ is automatically conserved if the geometry is flat. This is a consequence of the definition of $T^{a b}$ in Eq. (45) in terms of a potential. In analogy with elasticity theory, it is appropriate to think of $W$ as an Airy potential for $T^{a b}$ [11].

Note that the constraints break the invariance of the two dimensional bending energy with respect to conformal transformations of three-dimensional space. In general, the Gaussian curvature is not preserved. It is simple to show that under inversion in the origin $\mathbf{X} \rightarrow \mathbf{X} /|\mathbf{X}|^{2}$, one has [7]

$$
K_{\mathrm{G}} \rightarrow|\mathbf{X}|^{4} K_{\mathrm{G}}-2(\mathbf{X} \cdot \mathbf{n})|\mathbf{X}|^{2} K+4(\mathbf{X} \cdot \mathbf{n})^{2}
$$

Remarkably, the only geometries which remain flat are the cones we considered with apex at the origin, and which thus satisfy $\mathbf{X} \cdot \mathbf{n}=0$. Given one cone, its inversion in the origin is another [21]. Note, however, that curves on the unit sphere are invariant under inversion in the origin. Thus, while the physics might be different, the cone remains the same.

\section{B Cones as trajectories on spheres}

\section{B.1 Derivation of the Euler-Lagrange equations}

If we introduce the cutoff $r_{0}$ at the apex and integrate over the radial direction, the bending energy of a cone formed by a circular disc with its apex at the centre is given by

$$
H[\mathbf{u}]=\frac{1}{2} a \oint_{\Gamma} d s \kappa^{2}
$$


where $a$ is given by

$$
a=\ln \left(R / r_{0}\right) .
$$

The dimensional dependence is contained completely within this logarithm. This behavior is a consequence of the scale invariance of $H . H$ is a functional of curves on the unit sphere.

We have already pointed out in section 3.2 that if the boundary conditions are not symmetrical, the identification with Euler Elastica breaks down. It is, however, useful to see how the shape equation emerges from a variational principle which exploits the mapping from cones into trajectories on a sphere. Our task then is to identify the trajectories on a sphere minimizing the Hamiltonian $H$ given by Eq. (47).

Although the Euler-Lagrange equation itself is simple, its derivation is quite subtle. As we have found elsewhere, it is useful to introduce an appropriate set of auxiliary variables associated with local geometrical constraints [18]. Consider the functional

$$
F[\mathbf{u}, \mathbf{t}, \lambda, \Lambda, \mathbf{f}]=\oint_{\Gamma} d s\left[\frac{a}{2}\left(\mathbf{u} \cdot \mathbf{t} \times \mathbf{t}^{\prime}\right)^{2}+\frac{\lambda}{2}\left(\mathbf{u}^{2}-1\right)+\frac{\Lambda}{2}\left(\mathbf{t}^{2}-1\right)+\mathbf{f} \cdot\left(\mathbf{t}-\mathbf{u}^{\prime}\right)\right]
$$

where $\lambda(s)$ and $\Lambda(s)$ are two local Lagrange multipliers enforcing the constraints that $\mathbf{u}$ is a unit vector and that the parameter $s$ is arc-length on the sphere. The area constraint is thus implemented by fixing the range of integration on $s$ to the interval $[0,2 \pi]$.

Variation of $F$ with respect to $\mathbf{u}$ gives

$$
\mathbf{f}^{\prime}=-\left(a \kappa^{2}+\lambda\right) \mathbf{u}+a \kappa \mathbf{n}
$$

The right hand side of Eq. (50) has been simplified by using the structure equations (13) for the curve. Note, in particular, that $\mathbf{f}$ is not constant as it would be for a translationally invariant Hamiltonian. In the functional $F$ translational invariance is broken both in the energy itself and in the constraint on $\mathbf{u}$.

Variation with respect to $\mathbf{t}$ identifies the vector $\mathbf{f}$ appearing in Eq. (50) as

$$
\mathbf{f}=-\left(\Lambda+2 a \kappa^{2}\right) \mathbf{t}-a \kappa^{\prime} \mathbf{n},
$$

Later, we will provide a physical interpretation of $\mathbf{f}$ as an effective force per unit length along a ray of fixed $s$.

By substituting the expression for $\mathbf{f}$ given by Eq. (51) into Eq. (50) we obtain three equations describing the equilibrium. The projections along $\mathbf{u}$ and along $\mathbf{t}$ determine the multipliers $\lambda$ and $\Lambda$. The remaining equation along $\mathbf{n}$ is the Euler-Lagrange equation.

Note that

$$
\mathbf{f} \cdot \mathbf{u}=0 \text {. }
$$


Let us first examine the component of the conservation law along $\mathbf{t}$. We note that

$$
\mathbf{f}^{\prime} \cdot \mathbf{t} \stackrel{\sqrt[50]{=}}{=} 0 \text {. }
$$

This equation fixes the function $\Lambda$ appearing in Eq. (51) up to a constant. We have

$$
\Lambda=c-\frac{5 a}{2} \kappa^{2},
$$

where $c$ is a constant. We thus identify

$$
\mathbf{f}=\left(\frac{1}{2} a \kappa^{2}-c\right) \mathbf{t}-a \kappa^{\prime} \mathbf{n} .
$$

Comparing the two stress tensor projections (22) and (23) from Sec. 3.2 with the Lagrange multiplier $\mathbf{f}$ yields the remarkably simple relation $\mathbf{f}=a r^{2} \mathbf{f}_{\|}$if $T_{\| \perp}=0$. This confirms that, when this approximation is valid, $\mathbf{f}$ is the tangent stress along a ray of fixed $s$.

To determine $\lambda$, note that

$$
\mathbf{f}^{\prime} \cdot \mathbf{u} \stackrel{\sqrt[50]{=}}{=}-\left(a \kappa^{2}+\lambda\right) .
$$

Using Eq. (55), it follows that

$$
\lambda=-\frac{a}{2} \kappa^{2}-c .
$$

Note that this variable does not appear in the final shape equation which involves normal projections. For the record, we have

$$
\mathbf{f}^{\prime}=-\left(\frac{a}{2} \kappa^{2}-c\right) \mathbf{u}+a \kappa \mathbf{n} .
$$

Its normal projection together with Eq. (55) provides the Euler-Lagrange equation

$$
-a\left(\kappa^{\prime \prime}+\frac{1}{2} \kappa^{3}+\kappa\right)+c \kappa=0 .
$$

The constant $c$ is a tension associated with the fixed area constraint which is implied by fixing the arc-length. If this constraint is relaxed, $c=0$; however, there persists a 'tension' proportional to $a$ associated with constraining the curve trajectory to a sphere. Earlier we identified the origin of this term in the $r$ dependence of $\nabla^{2} K$ on the cone (see Sec. 3.2). We reproduce the shape equation (59) obtained earlier if we identify $C_{\|}$with the ratio of the constants $(c / a)$ (when $C_{\|}$is constant).

Generally, however, it appears to be beyond the scope of the variational analysis based on the reduced Hamiltonian to provide a consistent description of the cone. In particular, it does not appear to be possible to accommodate $T_{\| \perp} \neq 0$. Even when $T_{\| \perp}=0$, there is no obvious way, 
within this simplified framework, to analyze the $r$ dependent stresses set up in the cone as they, for instance, show up in the presence of gravity (see section 5).

We note that there is an integrability condition associated with Eq. (58). The identity $\oint d s \mathbf{f}^{\prime}=$ 0 associated with closure implies that the integrated source of $\mathbf{f}$ must also vanish. Thus

$$
\oint d s\left[\left(\kappa^{2}-2 c / a\right) \mathbf{u}-2 \kappa \mathbf{n}\right]=0 .
$$

This identity can be simplified further using Eq. (13b) to give

$$
\oint d s\left(\kappa^{2}-2 \sigma / a\right) \mathbf{u}=0 .
$$

There is no obvious physical interpretation of this identity within this one-dimensional framework. It is instructive to compare Eq. (61) with its analogue Eq. (29) within the general framework which we used to place a constraint on the stresses set up in the cone.

\section{B.2 Rotations and Conservation laws}

Fixing the apex of the cone at the origin breaks translational invariance. The residual symmetry is three-dimensional rotational invariance. The shape equation describing the sheet may be identified as the conservation law associated with this symmetry. In equilibrium, we must have

$$
\delta F=-\int d s \frac{d}{d s}[\mathbf{f} \cdot \delta \mathbf{u}+a \kappa(\mathbf{u} \times \mathbf{t}) \cdot \delta \mathbf{t}] .
$$

In particular, $\delta F=0$ under rotation. With $\delta \mathbf{u}=\mathbf{b} \times \mathbf{u}$ and $\delta \mathbf{t}=\mathbf{b} \times \mathbf{t}$, we have

$$
\mathbf{b} \cdot[\mathbf{u} \times \mathbf{f}+a \kappa \mathbf{u}]
$$

is constant. Thus

$$
\mathbf{u} \times \mathbf{f}+a \kappa \mathbf{u}=\mathbf{J},
$$

where $\mathbf{J}$ is a constant vector which can be identified as the tangential projection of the torque tensor (32)

$$
t_{a} \mathbf{m}^{a}=\mathbf{X} \times \mathbf{f}_{\|}+K \mathbf{u}=\mathbf{X} \times \frac{\mathbf{f}}{a r^{2}}+\frac{\kappa}{r} \mathbf{u}=\frac{1}{a r} \mathbf{J}
$$

if $T_{\| \perp}=0$.

Using the structure equations (13) and the Euler-Lagrange equation (59) one can easily show that $\mathbf{J}$ is indeed conserved. Squaring, using the identify $\mathbf{f} \cdot \mathbf{u}=0$, we find

$$
\mathbf{f}^{2}+a^{2} \kappa^{2}=\mathbf{J}^{2} .
$$

This is the first integral of the Euler-Lagrange equation. It can be rewritten

$$
\left(\kappa^{\prime}\right)^{2}+\frac{1}{4} \kappa^{4}-\frac{\sigma}{a} \kappa^{2}=\frac{\mathbf{J}^{2}-c^{2}}{a^{2}},
$$

and integrated after a separation of variables. 


\section{References}

[1] Hilbert D and Cohn-Vossen S Geometry and the Imagination (American Mathematical Society, Reprint edition 1999)

[2] Duncan JP and Duncan JL 1982 Proc. R. Soc. Lond. A 383191

[3] Fuchs D and Tabachnikov S 1999 Amer. Math. Monthly 10627

[4] Reference [3] has been updated and incorporated into a beautiful set of lectures in Fuchs D and Tabachnikov S Mathematical Omnibus: Thirty Lectures on Classic Mathematics (American Mathematical Society 2007)

[5] Struik D Lectures on Classical Differential Geometry (Dover Publications, 1988)

[6] Dal Co F, and Forster KW Frank O. Gehry: The Complete Works (Phaidon, 2003)

[7] Gray A Modern Differential Geometry of Curves and Surfaces with Mathematica (Chapman \& Hall/CRC, 2006)

[8] Lobhovsky A, Gentges S, Li H, Morse D and Witten T 1995 Science 2701482

[9] Ben Amar M and Pomeau Y 1997 Proc. R. Soc. Lond. A 453729

[10] Cerda E and Mahadevan L 2005 Proc. R. Soc. Lond. A 461671

[11] Landau LD and Lifschitz EM Theory of Elasticity, Third Edition (ButterworthHeinemann, 1997)

[12] Willmore TJ, Total Curvature in Riemannian Geometry (Chichester: Ellis Horwood, 1982)

[13] Canham P 1970 J. Theor. Biol. 26 61; Helfrich W 1973 Z. Naturforsch. C28 693

[14] Seifert U 1997 Adv. in Phys. 4613

[15] Cerda E, Mahadevan L and Pasini JM 2004 PNAS 1011806

[16] Let the surface be described parametrically by the mapping $\left(u^{1}, u^{2}\right) \rightarrow \mathbf{X}\left(u^{1}, u^{2}\right)$. The tangent vectors adapted to this parametrization are $\mathbf{e}_{a}=\partial_{a} \mathbf{X}, a=1,2$, and $\mathbf{n}$ is the unit normal. The induced metric on the surface and the extrinsic curvature are given by $g_{a b}=\mathbf{e}_{a} \cdot \mathbf{e}_{b}$ and $K_{a b}=\mathbf{e}_{a} \cdot \partial_{b} \mathbf{n}$ respectively [25, 26]. Indices are raised with the inverse metric $g^{a b}$. In terms of $K_{a b}$ and $g^{a b}$, the mean curvature and the Gaussian curvature are given respectively by $K=g^{a b} K_{a b}$ and $K_{\mathrm{G}}=\operatorname{det}\left(K_{a}^{b}\right)=\left(K^{2}-K^{a b} K_{a b}\right) / 2$.

[17] Capovilla R and Guven J 2002 J. Phys. A: Math. and Gen. 356233

[18] Guven J 2004 J. Phys. A: Math and Gen. 37 L313 
[19] Langer J and Singer D A 1984 Journ. Diff. Geom. 201

[20] Müller MM, Deserno M and Guven J 2005 Euro. Phys. Lett. 69 482; Phys. Rev. E 72 061407

[21] Castro-Villarreal P, Guven J and Vazquez P In preparation

[22] Müller MM, Deserno M, and Guven J 2007 Phys. Rev. E 76011921

[23] Starostin EL and van der Heijden GHM 2007 Nature Materials 6563

[24] Guven J, Müller MM and Vazquez P In preparation

[25] Montiel S and Ros A Curves and Surfaces (American Mathematical Society, 2005)

[26] Spivak M A Comprehensive Introduction to Differential Geometry. Vols. 1-5, Second Edition (Publish or Perish, 1979) 